Editorial

Eur J Trans Myol/Basic Appl Myol 2017; 27 (2): 77-80

\title{
Editorial 2017Spring PaduaMuscleDays, roots and byproducts
}

\author{
Ugo Carraro \\ IRCCS Fondazione Ospedale San Camillo, Venezia, Italy. \\ Email: ugo.carraro@ospedalesancamillo.net \\ This article is distributed under the terms of the Creative Commons Attribution Noncommercial License (CC BY-NC 4.0) which permits any \\ noncommercial use, distribution, and reproduction in any medium, provided the original author(s) and source are credited.
}

\begin{abstract}
The second 2017 issue of EJTM volume 27 contains the collection of abstracts from the 2017Spring PaduaMuscleDays conference, that was held March 23-25 in Montegrotto, Euganei Hills, Padova, Italy. In addition to a brief history of the Padova Myology Meetings held during the last 30 years, the present and the future of the PaduaMuscleDays conference are discussed with special reference to new media and the options they offer to spread to a larger audience the results of the many workshops held in the Hotel Augustus conference hall and in the Aula Guariento of the Accademia Galileiana di Scienze, Lettere ed Arti, one of the hidden treasures of the medioeval Padua, Italy. Preliminary announcements of the 2017 and 2018 events, in particular of the Giovanni Salviati Memorial, will follow.
\end{abstract}

Key Words: Translational Myology, PaduaMuscleDays, Basic and Applied Research in Myology

Eur J Trans Myol 2017; 27 (2): 77-80

The collection of abstracts from the 2017Spring PaduaMuscleDays (2017SpPMD) conference, that was held March 23 to 25 in Montegrotto, Euganei Hills (Padova) Italy, opens the second Issue of the European Journal of Translational Myology (EJTM) 27, 2017. ${ }^{1}$

The First Padua Myology Meeting was titled "Abano Terme Rehabilitation Conference", and focused, as the title suggests, on injury prevention combined with rehabilitation. These areas continue to be the driving interests which motivate a multidisciplinary audience to meet and interact during the PaduaMuscleDays (PMD). The first myology meeting was held in 1986 at a time during which implantable neuromuscular electrical stimulators seemed to be a very attractive potential treatment for spinal cord injury sequelae. Initially, meetings were held every three years, but because of the pressure from "Myology Friends" to meet more often, they became annual and then bi-annual events. Indeed, Spring and Autumn Meetings were held for several years. Thus, at this point, it is not easy to determine the number of Padua Myology Meetings that have been held during the last 30 years.

As interest in the meetings grew over time, the breadth of topics addressed by participants also increased. Muscle health and related injury prevention and rehabilitation are still a focal point; however, topics have also encompassed cardiac muscle work, including Dynamic Cardiomyoplasty which is based on the fascinating idea of supporting a weak heart by wrapping it within the latissimus dorsi skeletal muscle which is made to squeeze the ventricles synchronously by sensing and responding to the heart beat through electrical stimulation of its intramuscular nerve branches. ${ }^{2}$

In 1991, thanks to the support of Myology Friends, a new journal titled Basic and Applied Myology (BAM, retitled European Journal of Translational Myology, EJTM in 2010) began publishing original articles concerning biomedical research in myology and tentative applications of new knowledge to the prevention, cure and rehabilitation of mobility impaired young and old persons. Eventually, electrical stimulation of denervated muscle became the driving topic of the PaduaMuscleDays and this both nurtured and followed implementation of the EU Project RISE (Use of electrical stimulation to restore standing in paraplegics with long-term denervated degenerated muscles (QLG5-CT-2001-02191). ${ }^{3-8}$

Functional Electrical Stimulation was (and is), indeed, one of the main topics of the collective sessions, but in every event other issues related to muscle biology, imaging, function, disease and treatments were discussed. Many important science topics were covered and sometimes introduced. An important example of this includes the first world meeting in 1995 at Abano Terme (Padova), Italy which attracted the attention of molecular and cellular researchers and clinicians to the role of apoptosis in development, damage and repair of skeletal muscle and heart. ${ }^{9}$ This has become a very important research topic during the subsequent years. ${ }^{10}$ So given this history, it will not be surprising to find that the Collection of Abstracts from the 2017Spring PaduaMuscleDays covers new discoveries on the mechanisms of $\mathrm{Ca}^{2+}$ handling at the ultrastructural level, ${ }^{11}$ and spans to cover work on take-home rehabilitation strategies to rejuvenate older old humans. ${ }^{1,12}$ 


\section{Editorial}

Eur J Trans Myol/Basic Appl Myol 2017; 27 (2): 77-80

2017SpPMD Specifics

2017SpPMD Day One - Because it is a tradition of the PaduaMuscleDays to offer to young biomedical researchers and engineers the opportunity to hear the views and suggestions of Senior Myologists, Dirk Pette and Gerta Vrbova opened the 2017SpPMD by presenting a historical overview of their rodent experiments of daily low frequency neuromuscular stimulation that established the extent of plasticity of the different types of skeletal muscle fibers. In addition to their abstract, included in the Collection of 2017SpPMD, ${ }^{1}$ an updated review is published in EJTM 27 (1), 2017. ${ }^{13}$ We would further like to attract the attention of readers to the other interesting typescripts published in the same issue. ${ }^{14-17}$ It also contains the first paper of a new EJTM Section "Strength and Conditioning/Physical Exercise" edited by Antonio Paoli (Department of Biomedical Sciences, University of Padua, Italy), Pedro E. Alcarez (UCAM Research Center of High Performance Sport, San Antonio Catholic University of Murcia, Murcia, Spain), and Paulo Gentil (College of Physical Education and Dance, Federal University of Goias, Golania, Brazil). ${ }^{15}$

During the subsequent Workshop: "FES in mobility impairments", the present state of Clinical Functional Electrical Stimulation of muscle was discussed by Julien Gondin, Emiliana Bizzarini, Helmut Kern, Peter Biowski and Andrea Marcante.

2017SpPMD Day Two - The second day of the 2017SpPMD was dedicated to three topics. In the morning, Marco Sandri gave an excellent lecture on the underlying molecular mechanisms of muscle atrophy and sarcopenia. Then during the Workshop "Molecular Approaches on Interactions of Pain and Mobility in Elderly" presentations were given by Sandra Zampieri, Giovanna Albertin, Giulia Ottaviani, David Hood, Stefen Anton and Christiaan Leeuwenburgh. After the lunch in a sunny Piazza Duomo of Padova, the afternoon sessions were dedicated to the Workshop "Muscle Imaging" with presentations delivered by Feliciano Protasi, Amber Pond, ${ }^{18-20}$, Simona Boncompagni, Enrico Pierantozzi, Bert Blaauw, Paolo Gargiulo, Kyle J Edmunds, ${ }^{21,22}$ Andrea Marcante and Corrado Angelini. The lecture by Annalena Venneri "Physical exercise as a preventative strategy to slow down cognitive decline in aging. Evidence for the value of aerobic physical activity" closed the second day of the 2017SpPMD.

2017SpPMD Day Three: The morning of the third day of the 2017SpPMD was dedicated to the Workshop "Functional Rejuvenation in Aging." It started with the presentations of Jonathan Jarvis, Markus Gugatschka, Claus Gerstenberger, Matthias Leonhard and Fabian Volk. The Workshop: "Rehabilitation Strategies for Severe Muscle Atrophy and Dystrophy" was opened after lunch with a Lecture by Dario Coletti titled "Physical, pharmacological and nutritional interventions against muscle wasting and dystrophy." This was followed by presentations from Riccardo Ballarò, Daniela Tavian, Martin Schmoll, Polona Pečlin, Janez Rozman, and Thordur Helgason. ${ }^{23,24}$ The last session was dedicated to discussion of several interesting posters selected from the Poster Sessions and presented by Alfonc Baba, Laura Giaretta, Sergio Veneziani, Marina Marini. The presentation of the very interesting poster "The nutraceutical potential of Flaxseed bioactive compounds to treat muscular dystrophy" by Felicia Carotenuto ended the 2017SpPMD. ${ }^{25}$

You can learn more about the meeting by perusing the Abstracts, that include references and figures. ${ }^{1}$ Additional information is in the 2017SpPMD PROMO at: https://www.youtube.com/watch?v= NdbbeQFi9z8\&t=4s and in the recent Youtube Channel piece: https://www.youtube.com/channel/UCsh XMdDxpuOKa7eCWMBRqLg/videos?sort=dd\&view=0\&shelf_id=0

The conference is, indeed, expanding beyond the boundaries of time and walls to include the internet and social media. While the personal contacts that young researchers may have with experienced senior investigators is the main reason to organize the PaduaMuscleDays, those new opportunities for scientific interaction may be a second reason for experts in the many facets of Translational Myology to attend, offering easy-to-obtain valuable information to other investigators in both person and through cyberspace. The Youtube Channel is, and will be even more in the near future, filled with Promos of future meetings and with videos from the presentations.

Near future events will be the 3rd Padua Myology Seminar for general audiences, in particular of old persons, to be held September 21, 2017 in Padua's Accademia Galileiana di Scienze, Lettere ed Arti", that holds its meetings surrounded by the frescos of the Gothic painter, Guariento, who was the last before Giotto introduced the Italian Renaissance Style painting in the Scrovegni Chapel in Padua.

The 2018Spring PaduaMuscleDays will be held in Euganei Hills and Padua (Italy), March 14-16, 2018. The driving event will be the Symposium: "Giovanni Salviati Memorial" that will be held to honor a beloved friend and excellent scientist 20 years after his death. Colleagues and pupils will present their more recent results still influenced by the previous collaboration with Giovanni. Roger Sabbadini, Carlo Reggiani, Lars Larsson, Romeo Betto, Lee Sweeney, Massimo Zeviani, Paolo Bernardi, Marco Sandri, Leonardo Salviati, Clara Franzini-Armstrong, Feliciano Protasi, Paolo Gargiulo and Ugo Carraro 


\section{Editorial}

Eur J Trans Myol/Basic Appl Myol 2017; 27 (2): 77-80

accepted invitation. Rosario Rizzuto, Alfredo Margreth, Billi Di Mauro, Stefano Schiaffino, Sisto Luciani, Corrado Angelini, Paola Arslan and Anna Laverda Salviati are among the invited participants.

Suggestions of topics and speakers for other Workshops of the 2018SpPMD are welcomed.

\section{References}

1. Editors. Abstracts of the 2017Spring PaduaMuscleDays, Padua, Italy. Eur J Transl Myol 2017;27:81-112.

2. Carpentier A, Chachques JC. Myocardial substitution with a stimulated skeletal muscle: first successful clinical case. The Lancet 1985;325-8440:1267.

3. Kern H, Boncompagni S, Rossini K, et al. Long-term denervation in humans causes degeneration of both contractile and excitation- contraction coupling apparatus, which is reversible by functional electrical stimulation (FES). A role for myofiber regeneration? J Neuropathol Exp Neurol 2004;63:919-31.

4. Boncompagni S, Kern H, Rossini K, et al. Structural differentiation of skeletal muscle fibers in the absence of innervation in humans. Proc Natl Acad Sci U S A 2007;104:19339-44. Epub 2007 Nov 27.

5. Kern H, Carraro U, Adami N, et al. Home-based functional electrical stimulation rescues permanently denervated muscles in paraplegic patients with complete lower motor neuron lesion. Neurorehabil Neural Repair 2010;24:709-21.

6. Gargiulo P, Reynisson PJ, Helgason B, et al. Muscle, tendons, and bone: structural changes during denervation and FES treatment. Neurol Res 2011;33:750-8. doi: 10.1179/1743132811 Y.0000000007.

7. Carraro U, Kern H, Gava P, et al. Biology of Muscle Atrophy and of its Recovery by FES in Aging and Mobility Impairments: Roots and By-Products. Eur J Transl Myol 2015;25:221-30. doi: 10.4081/ejtm.2015.5272.

8. Kern H, Hofer C, Loefler S and al. Atrophy, ultra-structural disorders, severe atrophy and degeneration of denervated human muscle in SCI and Aging. Implications for their recovery by Functional Electrical Stimulation, updated 2017. Neurol Res 2017 Apr 13:1-7. doi: 10.1080/01616412.2017.1314906. [Epub ahead of print].

9. Carraro U, Franceschi C. Apoptosis of skeletal and cardiac muscles and physical exercise. Aging (Milano). 1997;9:19-34. Review.

10. Phaneuf S, Leeuwenburgh C. Apoptosis and exercise. Med Sci Sports Exerc 2001;33:393-6. Review.

11. Boncompagni S, Michelucci A, Pietrangelo, L et al. Discovery of new SR/TT junctions that mediate $\mathrm{Ca}^{2+}$ Entry in skeletal muscle. Eur J Transl Myol 2017;27:93.

12. Carraro U, Gava K, Baba A, Piccione F, Marcante A. Fighting muscle weakness in advanced aging by take-home strategies: Safe anti-aging full-body in-bed gym and functional electrical stimulation (FES) for mobility compromised elderly people. Biol Eng Med 2016;1:1-4. doi: 10.15761/BEM.1000106.

13. Pette d, Vrbová G. The contribution of neuromuscular stimulation in elucidating muscle plasticity revisited. Eur J Transl Myol 2017;27:33-39.

14. Pigna E, Greco E, Morozzi G, et al. Denervation does not induce muscle atrophy through oxidative stress. Eur J Transl Myol 2017;27:43-50.

15. Gentil P, Barbosa de Lira C, Paoli A, et al. Nutrition, pharmacological and training strategies adopted by six bodybuilders: case report and critical review. Eur J Transl Myol 2017;27:51-66.

16. Coletti D, Adamo S, Moresi V. Of faeces and sweat. How much a mouse is willing to run: having a hard time measuring spontaneous physical activity in different mouse sub-strains. Eur J Transl Myol 2017;27:67-70.

17. Power GA, Dalton BH, Gilmore KJ, et al. Maintaining motor units into old age: running the final common pathway. Eur J Transl Myol 2017;27:71-73.

18. Pond AL, Anderson LB, Cobb BA, et al. The ERG1 Potassium Channel is detected in human skeletal muscle with greater abundance in cachectic patients. Eur J Transl Myol 2017;27:94.

19. Pond A, Marcante A, Zanato R, et al. History, Mechanisms and Clinical Value of Fibrillation Analyses in Muscle Denervation and Reinnervation by Single Fiber Electromyography and Dynamic Echomyography. Eur J Transl Myol 2014;24:3297. doi: 10.4081/ejtm.2014.3297. eCollection 2014 Mar 31. 


\section{Editorial}

Eur J Trans Myol/Basic Appl Myol 2017; 27 (2): 77-80

20. Hockerman GH, Dethrow NM, Hameed S, et al. The Ubr2 gene is expressed in skeletal muscle atrophying as a result of hind limb suspension, but not Merg1a expression alone. Eur $\mathrm{J}$ of Transl Myol 2014;24:173-179. PubMed PMID: 25232465.

21. Edmunds KJ, Árnadóttir Í, Gíslason MK, et al. Nonlinear Trimodal Regression Analysis of Radiodensitometric Distributions to Quantify Sarcopenic and Sequelae Muscle Degeneration. Comput Math Methods Med 2016;2016:8932950. doi: 10.1155/2016/8932950. Epub 2016 Dec 27.

22. Edmunds KJ, Gíslason MK, Arnadottir ID, et al. Quantitative Computed Tomography and Image Analysis for Advanced Muscle Assessment. Eur J Transl Myol 2016;26:6015. doi: 10.4081/ejtm.2016.6015. eCollection 2016 Jun 13.

23. Helgason T, Arnason B, Gudmundsdottir V, et al. Comparing somatosensory evoked potentials resulting from transcutaneous spinal cord stimulation (tSCS) and tibial nerve stimulation in healthy and CP subjects. Eur J Transl Myol 2017;27:104.

24. Helgason T, Gunnlaugsdottir KI. Application of Acoustic-Electric Interaction for Neuro-Muscular Activity Mapping: A Review. Eur J Transl Myol 2015;24:4745. doi: 10.4081/ejtm.2014.4745. eCollection 2014 Nov 28. Review.

25. Carotenuto F, Costa A, Albertini MC, et al. Dietary Flaxseed Mitigates Impaired Skeletal Muscle Regeneration: in Vivo, in Vitro and in Silico Studies. Int J Med Sci 2016;13:206-19. doi: 10.7150/ijms. 13268 . 